\title{
Hall coefficient and ARPES in systems with strong pair fluctuations
}

\author{
Alfonso Romano ${ }^{(a)}$ and Julius Ranninger ${ }^{(b)}$ \\ (a) Dipartimento di Scienze Fisiche "E.R. Caianiello", Università di Salerno, I-84081 Baronissi (Salerno), Italy \\ Unità I.N.F.M. di Salerno \\ (b) Centre de Recherches sur les Très Basses Températures, Laboratoire Associé à l'Université Joseph Fourier, \\ Centre National de la Recherche Scientifique, BP 166, 38042, Grenoble Cédex 9, France
}

(October 31, 2018)

\begin{abstract}
We examine the normal-state temperature and doping dependence of the Hall coefficient in the context of a pair-fluctuation scenario, based on a model where itinerant electrons are hybridized with localized electron pairs via a charge exchange term. We show that an anomalous behavior of the Hall effect, qualitatively similar to that observed in high- $T_{c}$ superconductors, can be attributed to the non-Fermi liquid properties of the single-particle spectral function which exhibits pseudogap features. Our calculations are based on a dynamical mean-field procedure which relates the transport coefficients to the single-particle spectral function in an exact way.

PACS numbers: 72.15.Gd, 74.25.-q, 74.25.Jb, 79.60.-i
\end{abstract}

\section{INTRODUCTION}

The anomalous temperature and doping behavior of transport coefficients [1] such as the dc resistivity $\rho$, the Hall coefficient $R_{H}$, the magnetoresistance $\Delta \rho$ and the Hall angle $\cot \theta_{H}=\rho / R_{H}$ in the normal state of high- $T_{c}$ cuprate superconductors (HTS) are amongst the clearest indications for non-Fermi liquid behavior in these materials. Quite generally $R_{H}$ shows a rapid decrease with increasing temperature and in certain overdoped samples can change from positive to small negative values. This, together with the Hall angle showing an unexpected quadratic temperature variation in the underdoped regime, has led to speculations about different transport mechanisms in presence and in absence of a magnetic field [2], based on the phenomenological introduction of two different scattering rates - perpendicular and parallel to the Fermi surface. Such a description may hold in non-Fermi liquids, provided they retain sufficient Fermi liquid properties so that the concept of scattering rates and a description in terms of a Boltzmann equation is still meaningful. When this is not guaranteed, the interpretation of the anomalous transport properties has to be sought in the single-particle spectral features themselves, strongly affected by many-body effects. The Mott-Hubbard scenario of a strongly correlated system [3,4] as well as the $t-J$ spin correlation picture [5], which are believed to capture certain features of the underdoped HTS, have been investigated in this connection and show qualitative similarities with the experimental results.

We shall in this Letter address the question of the anomalous magneto-transport processes in terms of a scenario where the Fermi liquid properties get destroyed in the normal state due to dynamical electron pair formation showing up in the appearence of a pseudogap. The decrease of $R_{H}$ with increasing temperature is then expected to be related to thermal excitations across such a pseudogap feature in the single-particle spectra. The present study aims to examine the underdoped and the overdoped systems on equal footing.

It is presently a matter of debate whether the onset of the pseudogap at $T^{*}$ is a precursor of the superconducting state or not. If not, pair fluctuations are a necessary but not a sufficient condition for the system to become superconducting upon lowering the temperature. The present experimental situation seems to suggest that the opening of the pseudogap is not triggered by superconducting precursor effects. If it were, then superconducting correlations should show up in the entire temperature regime of the pseudogap phase, giving rise to an enhancement of the dc conductivity, the diamagnetism etc. into a Meissner state. Such features are indeed observed, but only in a very reduced fraction of the pseudogap temperature regime relatively close to $T_{c}$, where such pair fluctuations exist on a time scale of the order of $T_{c}$. Recent experiments on the frequency dependence of the Meissner screening [6] seem also to confirm that.

Presently there are two phenomenological models, the negative-U Hubbard model and the Boson-Fermion model (BFM), which are studied in connection with those experimental findings. In the negative-U Hubbard model the HTS physics is treated as a cross-over phenomenon between a BCS state in the overdoped regime and something close to a Bose-Einstein condensation (BEC) of preformed pairs in the underdoped regime [7]. Going from one extreme doping situation to the other one is monitored by a variation of the attractive interaction $\mathrm{U}$ between the electrons. In the BFM the HTS physics is described by a mechanism by which pairing in an electronic subsystem is induced by resonant scattering of the electrons in and out of localized tightly bound pair electron (bosonic) states. Doping is monitored by changing the position of the bosonic energy level which alters the relative occupation between the electrons and the bosons. The bosonic states in the BFM have their counterpart in 
the negative- $U$ Hubbard system in form of two-particle resonant states above the chemical potential.

The BFM was originally introduced [8] and studied in some detail in the past as regards the single-particle spectral properties of the electrons [9]. The qualitative differences which exist between the underdoped and the overdoped regime warrant to examine whether and how these differences can possibly be held responsible for the unusual temperature and doping dependence of certain magneto-transport properties seen in the HTS. Such a study must be done in a way which relates the above mentioned transport coefficients to the single-particle properties in a rigorous fashion. In the limit of infinite dimensions this can be achieved within the dynamical meanfield theory approach which we shall adopt here.

The hamiltonian for the BFM is given by

$$
\begin{aligned}
H= & \varepsilon_{0} \sum_{i, \sigma} c_{i \sigma}^{\dagger} c_{i \sigma}-t \sum_{\langle i j\rangle, \sigma} c_{i \sigma}^{\dagger} c_{j \sigma} \\
& +E_{0} \sum_{i} b_{i}^{\dagger} b_{i}+g \sum_{i}\left[b_{i}^{\dagger} c_{i \downarrow} c_{i \uparrow}+c_{i \uparrow}^{\dagger} c_{i \downarrow}^{\dagger} b_{i}\right] .
\end{aligned}
$$

$c_{i \sigma}^{(\dagger)}$ denote annihilation (creation) operators for electrons with spin $\sigma$ at some effective sites $i$ (involving molecular units rather than individual atoms) and $b_{i}^{(\dagger)}$ denote hardcore bosonic operators describing tightly bound electron pairs. $t, D, \Delta_{B}$ and $g$ denote respectively the bare hopping integral for the electrons, the bare electronic half bandwidth, the boson energy level and the boson-fermion pair-exchange coupling constant. Furthermore we put $\varepsilon_{0}=D-\mu$ and $E_{0}=\Delta_{B}-2 \mu$ and assume the chemical potential $\mu$ to be common to fermions and bosons (up to a factor 2 for the bosons) in order to guarantee charge conservation. These parameters are fixed in such a way that at temperatures large compared to the interaction $g$ the number of fermions $n_{F}=\sum_{i, \sigma}\left\langle c_{i \sigma}^{\dagger} c_{i \sigma}\right\rangle$ lies in the interval $[1,0.75]$ which covers the typical experimental doping regime. We furthermore choose $n=n_{F}+2 n_{B}$ $\left(n_{B}=\frac{1}{N} \sum_{i}\left\langle b_{i}^{\dagger} b_{i}\right\rangle\right.$ denoting the number of bosonic electron pairs) to lie in the interval $[1,2]$ in order to account for the appearence of a pseudogap phase. For the present analysis we choose $n=1.5$ as a representative value, having verified that in the regime $n=[1.2,1.8]$ the various physical quantities which we shall discuss here exhibit qualitatively the same behavior. Finally, in order to have a $T^{*}$ of the order of a few hundred degrees $\mathrm{K}$ we choose $g=0.2$ (all energies are in units of the bare electronic bandwidth $2 D$ ). From our previous studies we know that the anomalous electronic properties in the normal state are largely determined by those on an atomic or molecular level 10. The dynamical mean-field approach permits to properly deal with the local electronic structure which is renormalized by the itinerancy of the electrons via a Weiss mean-field 11 .

\section{ON THE ORIGIN OF THE PSEUDOGAP}

In order to highlight the physics leading up to the pseudogap phenomenon, let us first consider the above Hamiltonian in the atomic limit i.e., $t_{i j}=0$ [10]. In that case we derive the following set of eigenstates $|n\rangle$ and eigenvalues $E_{n}$ :

$$
\begin{aligned}
|1\rangle & =|0\rangle, & E_{1} & =0 \\
|2\rangle & =|\uparrow\rangle, & E_{2} & =\varepsilon_{0} \\
|3\rangle & =|\downarrow\rangle, & E_{3} & =\varepsilon_{0} \\
|4\rangle & =u|\uparrow \downarrow\rangle-v|\bullet\rangle, & E_{4} & =\varepsilon_{0}+E_{0} / 2-\gamma \\
|5\rangle & =v|\uparrow \downarrow\rangle+u|\bullet\rangle, & E_{5} & =\varepsilon_{0}+E_{0} / 2+\gamma \\
|6\rangle & =|\uparrow \bullet\rangle, & E_{6} & =\varepsilon_{0}+E_{0} \\
|7\rangle & =|\downarrow \bullet\rangle, & E_{7} & =\varepsilon_{0}+E_{0} \\
|8\rangle & =|\uparrow \downarrow \bullet\rangle, & E_{8} & =2 \varepsilon_{0}+E_{0}
\end{aligned}
$$

The presence on a given site of an electron with spin up and down, respectively, is denoted by corresponding arrows and the presence of a Boson is denoted by a dot. Moreover we have

$$
\begin{gathered}
u^{2}=\frac{1}{2}\left[1-\frac{\varepsilon_{0}-E_{0} / 2}{\gamma}\right], v^{2}=\frac{1}{2}\left[1+\frac{\varepsilon_{0}-E_{0} / 2}{\gamma}\right] \\
\gamma=\left[\left(\varepsilon_{0}-E_{0} / 2\right)^{2}+g^{2}\right]^{1 / 2}, \quad u v=\frac{g}{2 \gamma}
\end{gathered}
$$

The corresponding atomic Green's function takes the form

$$
\begin{array}{r}
G_{0}\left(i \omega_{n}\right)=-\int_{0}^{\beta} d \tau e^{i \omega_{n} \tau}<T\left[c_{\uparrow}(\tau) c_{\uparrow}^{\dagger}\right]>= \\
\frac{Z_{f}}{i \omega_{n}-\varepsilon_{0}}+\left(1-Z_{f}\right)\left[\frac{u^{2}}{i \omega_{n}-E_{4}+\varepsilon_{0}}+\frac{v^{2}}{i \omega_{n}-E_{5}+\varepsilon_{0}}\right]
\end{array}
$$

where the spectral weight of the non-bonding singleparticle excitations is given by

$$
Z_{f}=\frac{1}{Z_{0}}\left(1+e^{-\beta \varepsilon_{0}}+e^{-\beta\left(\varepsilon_{0}+E_{0}\right)}+e^{-\beta\left(2 \varepsilon_{0}+E_{0}\right)}\right)
$$

and $Z_{0}=\sum_{n} \exp \left(-E_{n} / k_{B} T\right)$ denotes the partition function. The spectral weights for the bonding and antibonding two-particle states are given by $\left(1-Z_{f}\right) u^{2}$ and $\left(1-Z_{f}\right) v^{2}$, respectively. We stress that the form of $G_{0}\left(i \omega_{n}\right)$ is formally equivalent to that of a BCS Green's function, where $g^{2}$ plays the role of the usual gap function, which, of course, in this lowest order approximation does not depend on temperature. $Z_{f}$ in general decreases with decreasing temperature, which is the crucial effect that controls the pseudogap physics in this model. It leads, already on a purely local electronic consideration, to a situation where the spectral weight of the singleparticle states diminishes with decreasing temperature, 
while at the same time the spectral weight of the twoparticle bonding and antibonding states increases. These features are reminiscent of the situation of a BCS superconductor below $T_{c}$ where the role of the two-particle states is played by the Cooper pairs.

\section{DYNAMICAL MEAN FIELD THEORY APPROACH TO THE BFM}

In developing a theoretical description which accounts for the itinerancy of the electrons, care must be taken in fully taking into account the local electronic structure which contains the essential ingredients of the pseudogap phase. This can be done by using as a starting basis the atomic limit of the model and then introducing the effects of the electron itinerancy within the Dynamical Mean Field Theory (DMFT) 11]. In this approach a many-body system is seen as a purely local system coupled to a "medium", representing a Weiss field to be determined self-consistently. The problem is cast into the form of a single-impurity Anderson problem described by an effective Hamiltonian

$$
\begin{aligned}
H= & \sum_{\sigma} \varepsilon_{0} c_{\sigma}^{\dagger} c_{\sigma}+E_{0} b^{\dagger} b+g\left[c_{\uparrow}^{\dagger} c_{\downarrow}^{\dagger} b+b^{\dagger} c_{\downarrow} c_{\uparrow}\right] \\
& +\sum_{k, \sigma} w_{k} d_{k \sigma}^{\dagger} d_{k \sigma}+\sum_{k, \sigma} v_{k}\left[d_{k \sigma}^{\dagger} c_{\sigma}+c_{\sigma}^{\dagger} d_{k \sigma}\right]
\end{aligned}
$$

where $c_{\sigma}^{(\dagger)}$ and $b^{(\dagger)}$ denote the original fermion and boson operators, respectively, at a single impurity site and $d_{k \sigma}^{(\dagger)}$ denote the auxiliary Fermionic operators associated with the Weiss field. The evaluation of their energy spectrum $w_{k}$ and their coupling $v_{k}$ to the impurity electrons has to be performed in a self-consistent way.

It can be shown that the impurity Green's function can be cast into the form

$$
G_{i m p}\left(\omega_{n}\right)=\frac{1}{i \omega_{n}-\varepsilon_{0}-\Sigma_{W}\left(\omega_{n}\right)-\Sigma_{i n t}\left(\omega_{n}\right)}
$$

which makes explicit two momentum-independent contributions to the self-energy: one contribution being due to the boson-fermion exchange coupling and denoted by $\Sigma_{\text {int }}(\omega)$, and one contribution being due to the hybridization of the impurity center with the medium and denoted by $\Sigma_{W}(\omega)$. This latter quantity, depending on the parameters $v_{k}$ and $w_{k}$ and generally referred to as the Weiss self-energy, is determined self-consistently by equating the impurity Green's function (7) to the integral over the $\mathbf{k}$ space of the lattice Green's function

$$
G_{l a t}\left(\omega_{n}, \varepsilon_{\mathbf{k}}\right)=\frac{1}{i \omega_{n}+\mu-\varepsilon_{\mathbf{k}}-\Sigma_{i n t}\left(\omega_{n}\right)}
$$

where $\varepsilon_{\mathbf{k}}$ denotes the bare electron dispersion. Upon replacement of the integration over $\mathbf{k}$ by an integration over energy, the integrated lattice Green's function takes the form

$$
G_{l a t}\left(\omega_{n}\right)=\int d \varepsilon \frac{\rho(\varepsilon)}{i \omega_{n}+\mu-\varepsilon-\Sigma_{\text {int }}\left(\omega_{n}\right)} .
$$

Assuming, as is usually done, a Bethe lattice in infinite dimensions, the density of states (DOS) for the bare electrons appearing in eq.(9) is the semi-circular DOS $\rho(\varepsilon)=\left(1 / 2 \pi t^{2}\right) \sqrt{\varepsilon(4 t-\varepsilon)}$ of width $2 D=4 t$. The selfconsistency condition $G_{i m p}\left(\omega_{n}\right)=G_{l a t}\left(\omega_{n}\right)$ implies

$$
\Sigma_{W}\left(\omega_{n}\right)=t^{2} G_{i m p}\left(\omega_{n}\right)
$$

or alternatively

$$
\Delta(\omega)=t^{2} A_{F}(\omega)
$$

where $A_{F}(\omega)=-2 \operatorname{Im} G_{i m p}\left(\omega_{n}=\omega+i \delta\right)$ is the fermionic DOS and

$$
\Delta(\omega)=2 \pi \sum_{k} v_{k}^{2} \delta\left(\omega-w_{k}\right)
$$

is the spectral function associated with the self-energy of the auxiliary fermions.

The above impurity problem is solved here within the so-called Non Crossing Approximation (NCA) [12], along the lines presented and discussed in Ref. [13]. Within the DMFT framework, NCA has recently been applied to several other models, such as a multiband Hubbard model for perovskites [14], the Anderson lattice model with correlated conduction electrons [15] and the Kondo lattice model with correlated conduction electrons [16].

One serious drawback of the DMFT $+\mathrm{NCA}$ procedure is that it does not recover the non-interacting limit, corresponding in the BFM to the case $g=0$. The point is that this approach leads to some kinematic interactions which result in a self-energy $\Sigma_{\text {int }}(\omega)$ which does not vanish as the coupling constant $g$ tends to zero. In this section we highlight this shortcoming for the fully symmetric case, realized when the boson site energy and the chemical potential are both pinned in the middle of the fermionic band, giving $n=2$ for all temperatures. In the top figure of Fig.1 we plot $\operatorname{Im} \Sigma_{\text {int }}(\omega)$ at $T=0.04$ for different coupling constants $g$, as obtained within the conventional NCA approach. We notice a strong dependence on $g$ for the frequency regime around the chemical potential, characterized by a rapid decrease as $g$ tends to zero, as it should be. On the contrary, for larger frequencies $\operatorname{Im} \Sigma_{\text {int }}(\omega)$ is sizeable and shows little dependence on the value of $g$. This fact indicates the existence of kinematical interactions which, contrary to what one would expect, are effective even for $g=0$ and thus should be subtracted out in the calculation procedure. In order to do that, we first consider the case without interaction $(g=0)$ for a given temperature and calculate selfconsistently the interaction self-energy $\Sigma_{\text {int }}^{g=0}(\omega)$ within the standard DMFT+NCA approach. As just pointed 
out, this leads to an unphysical expression which over a large range of energies does not vanish, as it ought to in the absence of interaction. We then repeat the whole calculation for finite boson-fermion coupling at the same temperature, obtaining a given expression $\Sigma_{\text {int }}(\omega)$ for the interaction self-energy. Finally, we replace in the integrated lattice Green's function (9) the self-energy $\Sigma_{\text {int }}(\omega)$ calculated at finite $g$ by $\Sigma_{\text {int }}(\omega)-\Sigma_{\text {int }}^{g=0}(\omega)$, thus subtracting out the unphysical part of the interaction self-energy coming from the non-interacting case. In this way eq. (9) is rewritten in the form

$$
G_{\text {lat }}^{*}(\omega)=\int d \varepsilon \frac{\rho(\varepsilon)}{\omega+\mu-\varepsilon-\Sigma_{\text {int }}(\omega)+\Sigma_{\text {int }}^{g=0}(\omega)},
$$

thus leading to a redefined integrated lattice Green's function which in the non-interacting case correctly gives back the fermionic DOS $\rho(\varepsilon)$ for free electrons.

In the bottom panel of Fig.1 we illustrate the behavior of $\operatorname{Im} \Sigma_{i n t}(\omega)$ as evaluated within this redefined NCA approach. From a comparison with the curves reported in the upper panel, we notice that as far as the low frequency behavior around the chemical potential is concerned, the two methods give results which are in better and better agreement as increasing values of $g$ are considered. Outside this frequency regime the self-energy is in general small when evaluated within the redefined NCA, going to zero for $g=0$, as it actually ought to be the case.
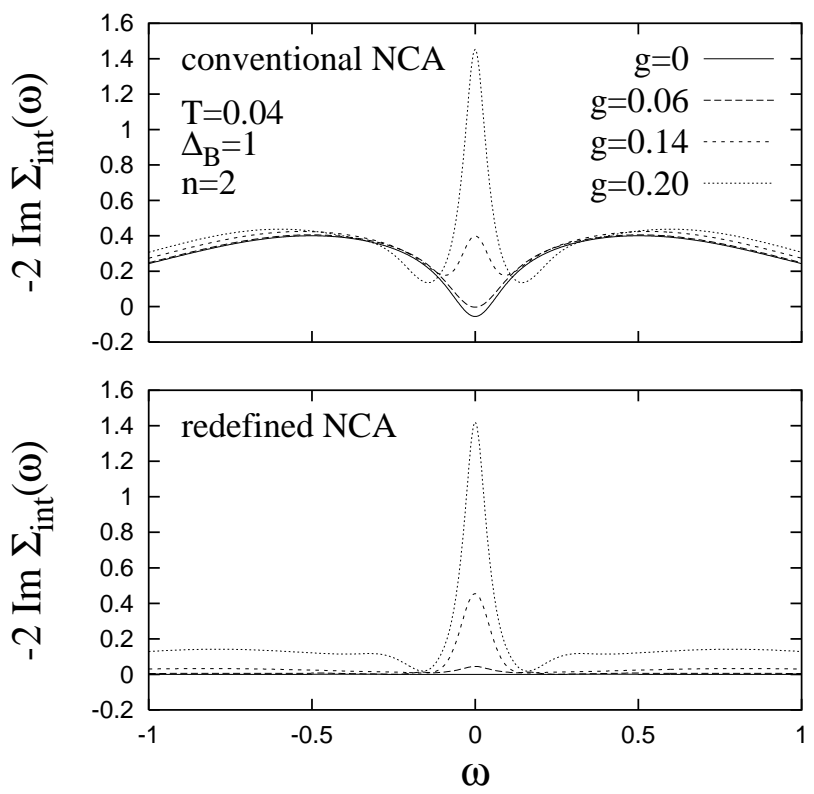

FIG. 1. Comparison in the fully symmetric case $\left(\Delta_{B}=1\right.$ and $n=2$ ) of the imaginary part of the interaction self-energy evaluated within the conventional NCA (top panel) and the redefined NCA approach (bottom panel) for various coupling constants $g$ and $T=0.04$

This difference in the self-energy also affects the be- havior of the density of states. In Fig.2 we compare the fermionic DOS evaluated within the conventional NCA, given by $A_{F}(\omega)=-2 \operatorname{Im} G_{\text {lat }}(\omega)$, with that evaluated within the redefined NCA approach, given by $A_{F}^{*}(\omega)=$ $-2 \operatorname{Im} G_{l a t}^{*}(\omega)$. We again notice that for small values of $g$ the discrepancy between the two approaches is noticeable, with unphysical tails in the DOS obtained from the standard NCA which should not be present. As higher values of the coupling $g$ are considered, the agreement between the two NCA approaches is increasingly improved, in particular in the frequency regime close to the chemical potential.

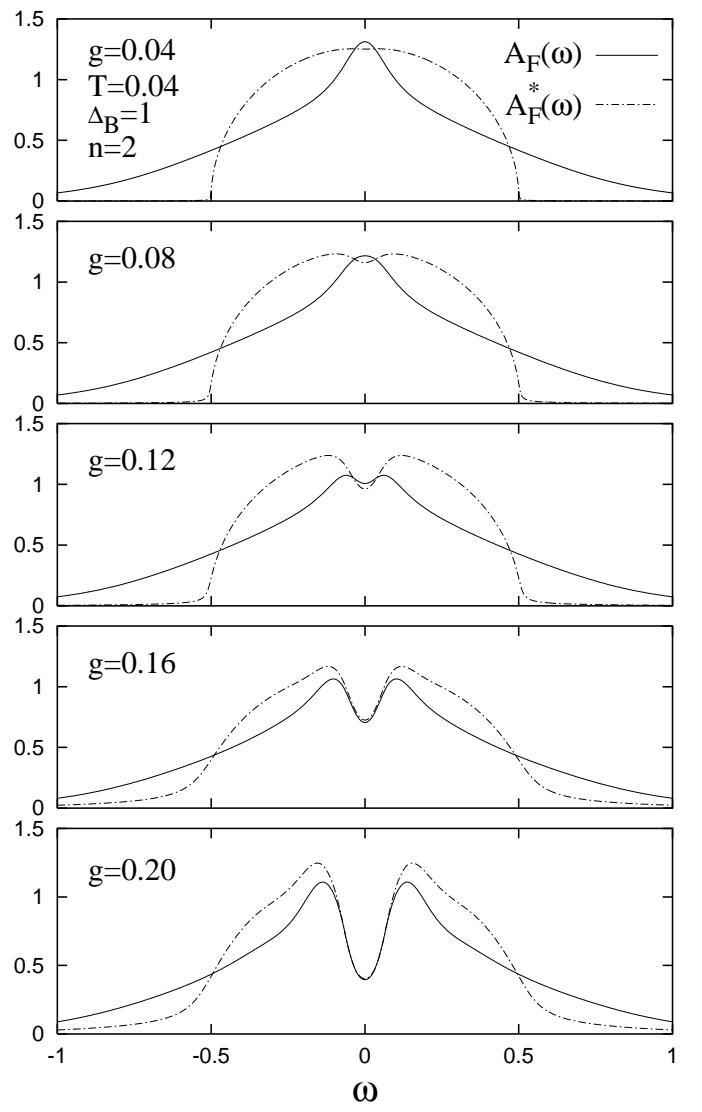

FIG. 2. Comparison in the fully symmetric case $\left(\Delta_{B}=1\right.$ and $n=2)$ of the fermionic DOS $A_{F}(\omega)$ and $A_{F}^{*}(\omega)$, evaluated respectively within the conventional and the redefined NCA approach for various coupling constants $g$ and $T=0.04$

The situation away from the fully symmetric case is numerically more involved. We have nonetheless verified that the results obtained in a number of specific cases for $n \neq 2$ are in agreement with our findings for the fully symmetric case. Since here we are interested in analyzing cases in which the hybridization constant $g$ is of the order of 0.2 , we conclude that we can safely work with the conventional NCA approach, which in this regime is expected to give correct results. This will be done for a variety of cases in the next sections. In particular, our 
aim is to evaluate the single-particle fermionic Green's function from which measureable quantities can be derived, such as the angle-resolved direct and inverse photoemission spectra (ARPES and ARIPES, respectively), and certain transport coefficients for which vertex corrections, in the limit of infinite dimensions, can be neglected.

\section{THE ARPES AND INVERSE ARPES SPECTRUM}

We examine first of all the doping and temperature dependence of the spectral function of the lattice Green's function for fermions $A_{F}\left(\varepsilon_{\mathbf{k}}, \omega\right)=-2 \operatorname{Im} G_{\text {lat }}\left(\varepsilon_{\mathbf{k}}, \omega\right)$. Within the BFM scenario the doping process is primarily controlled by a variation of the position of the bosonic level. For a given $n$ this implies a relative change in concentration between the bosons and fermions which leads to a reduction of $n_{F}$ with decreasing $\Delta_{B}$. We should stress that doping in HTS is an extremely complicated process which involves charge transfer from the dielectric layers into the conduction plane. Monitoring the doping process by changing the position of the Bosonic energy level represents only a crude mechanism for it (though the most significant one for this model). Other features such as anisotropy effects and electronic correlations of course play a role, but are neglected here.

The temperature and doping dependence of the singleparticle electron spectral function $A_{F}\left(\varepsilon_{k}, \omega\right)$ is studied for $\varepsilon_{k}=\varepsilon_{k_{F}}$, where $\varepsilon_{k_{F}}$ is determined from the condition that the distribution function $n_{F}\left(\varepsilon_{k}\right)=\int d \omega A_{F}\left(\varepsilon_{k}, \omega\right)$ is independent on the temperature [17]. The behavior of $A_{F}\left(\varepsilon_{k_{F}}, \omega\right)$ is easily interpreted in terms of the level spectrum of the BFM in the atomic limit [10]. As demonstrated in Section 3, this spectrum consists of bonding and antibonding two-particle states having energies $E_{4}$ and $E_{5}$, plus a non-bonding single-particle state having energy $\varepsilon_{0}$. The dynamical mean field, mimicking the electron itinerancy, broadens these levels into a continuous spectrum with peaks approximately centered around $\varepsilon_{-}=E_{4}-\varepsilon_{0}, \varepsilon_{+}=E_{5}-\varepsilon_{0}$ and $\varepsilon_{0}$ (see Fig.3). We notice that there is a qualitative difference between the underdoped $\left(\Delta_{B}=1.2\right)$ and the overdoped $\left(\Delta_{B}=1\right)$ situation (here $\Delta_{B} / 2$ gives the position of the energy level of the two-particle bosonic state with respect to the bare electronic band, as measured from its bottom). In the case of an underdoped system we can clearly distinguish spectral features (Fig.3a) with a predominant peak corresponding to the non-bonding state which, as the temperature decreases, has its intensity transferred to the lower-lying two-particle bonding state and thereby leads to the opening of a pseudogap around the chemical potential. This is quite different from what happens in the overdoped situation (Fig.3b) where the spectral weight is roughly equally distributed between the bonding and the antibonding two-particle states and no clear feature for the opening of a pseudogap is visible. This effect is particularly evident when plotting (Fig.4) the spectral function at low temperature, together with the corresponding intensity for Angle-Resolved Photoemission Spectroscopy (ARPES) given by $A_{F}\left(\varepsilon_{k_{F}}, \omega\right) f(\omega)(f(\omega)$ denoting the Fermi function) for different doping levels (different values of $\Delta_{B}$ ). The results in Fig. $4 \mathrm{~b}$ are in good agreement with the experimental ARPES indication of a pseudogap, well opened in the underdoped regime and practically not visible in the overdoped one. In Fig.4c we plot the intensity for Angle-Resolved Inverse Photoemission Spectroscopy (ARIPES) given by $A_{F}\left(\varepsilon_{k_{F}}, \omega\right)(1-f(\omega))$ which clearly indicates the contribution from the antibonding two-particle states above the chemical potential. With the rapid improvement of photemission spectroscopy experiments such a feature might be resolved in some near future.

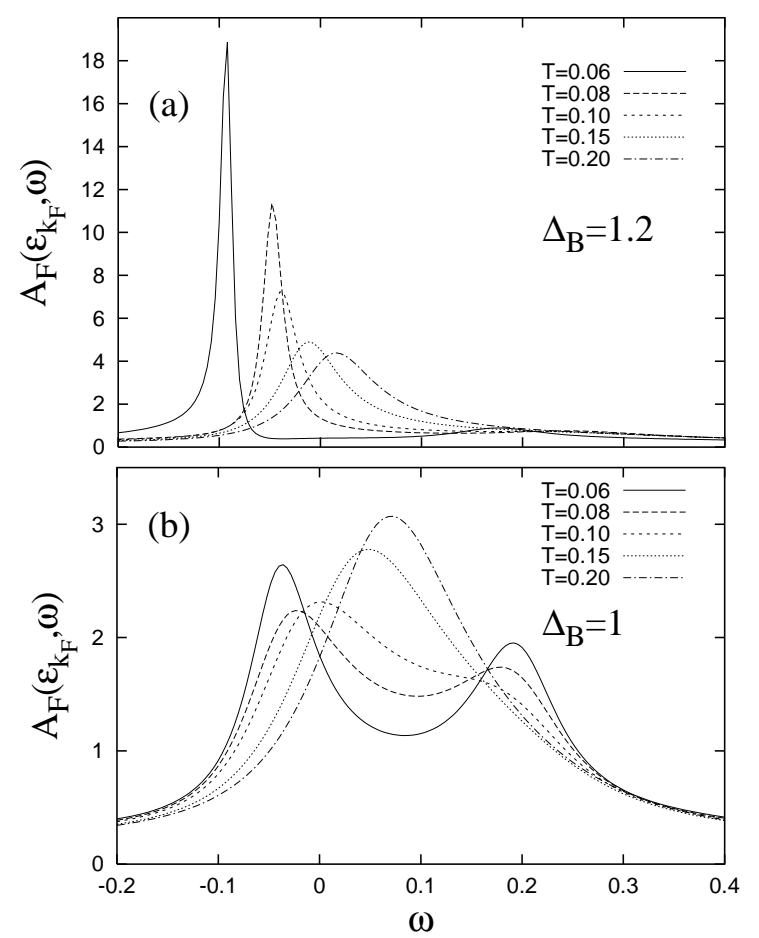

FIG. 3. The electron spectral function at the Fermi surface in the underdoped (a) and the overdoped (b) case for $g=0.2$ and $n=1.5$ (energies plotted with respect to the chemical potential)

Let us now turn to the discussion of the ordinary dc $(\sigma)$ and Hall $\left(\sigma_{H}\right)$ conductivity and show to what extent they are influenced by the anomalous single-particle spectral properties discussed above. In the limit of infinite dimensionality these quantities reduce to [3, 4 ]

$$
\begin{array}{r}
\sigma=\frac{\pi e^{2}}{2 d} \int d \varepsilon \rho(\varepsilon) \int d \omega\left(-\frac{\partial f(\omega)}{\partial \omega}\right) A_{F}(\varepsilon, \omega)^{2} \\
\sigma_{H}=\frac{\pi^{2} e^{3} H}{3 d^{2}} \int d \varepsilon \varepsilon \rho(\varepsilon) \int d \omega\left(-\frac{\partial f(\omega)}{\partial \omega}\right) A_{F}(\varepsilon, \omega)^{3} .
\end{array}
$$


From the above expressions we evaluate, with the help of the single-particle spectral functions $A_{F}(\varepsilon, \omega)$ determined before, the temperature and doping dependence of the Hall coefficient $R_{H}=\sigma_{H} / \sigma^{2} H$. In Fig.5 we plot $R_{H}$ as a function of temperature for different doping levels. As we decrease $\Delta_{B}$ for a fixed temperature, $R_{H}$ decreases, showing a sign change in the high-temperature regime below $T \simeq 0.17$. In the underdoped regime $\left(\Delta_{B} \simeq 1.2\right) R_{H}$ increases rapidly as the temperature decreases, while a similar temperature variation in the overdoped limit $\left(\Delta_{B} \simeq 1\right)$ changes $R_{H}$ only moderately. With decreased doping (increasing $\Delta_{B}$ ) the temperature at which the sign change occurs moves upwards. The sign change of $R_{H}$ suggests a change-over from negative charge carriers at high temperatures to positive charge carriers at low temperature. While at high temperatures $R_{H}$ is roughly determined by the concentration of electrons $n_{F}$, at low temperatures on the contrary it is roughly controlled by $1-n_{F}$ and hence scales with doping.
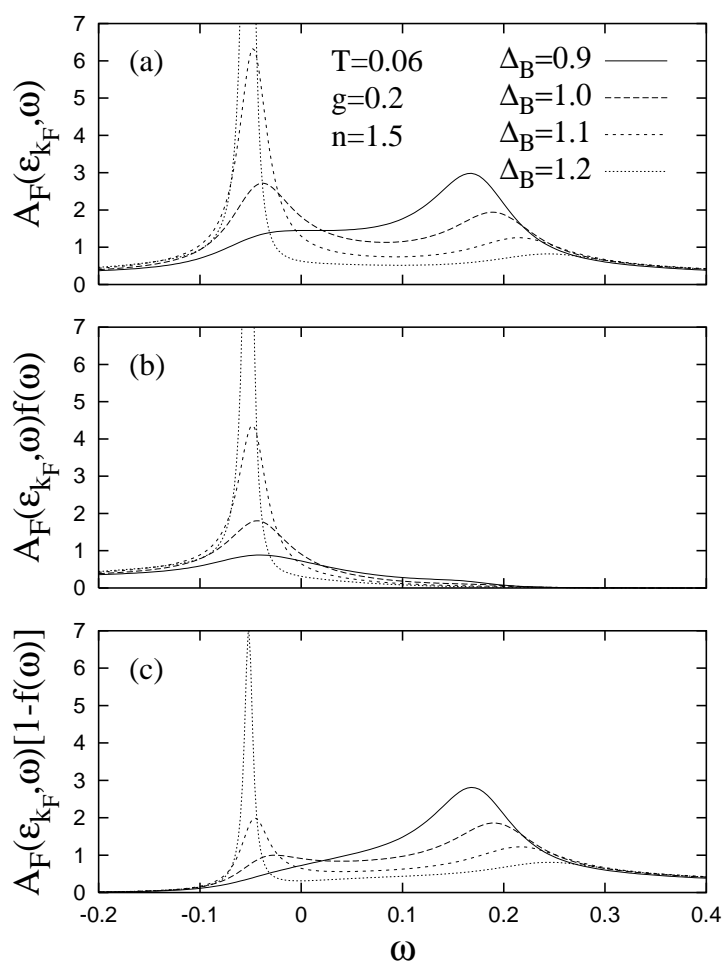

FIG. 4. The low-temperature electron spectral function at the Fermi surface (a) and the corresponding ARPES (b) and ARIPES (c) intensities for $n=1.5, g=0.2$ and different doping levels (different $\Delta_{B}$ ). The energies are measured relative to the chemical potential.

Although experiments seem to suggest that for underdoped systems $R_{H}$ is always positive, our results indicate that as doping is reduced the sign change occurs for higher and higher temperatures, possibly not reachable in real experiments. At first sight it is tempting to inter- prete the results of Fig.5 as a change-over from a large Fermi surface at high temperatures to small Fermi surface pockets at low temperatures. However, our study of the single-particle spectral function indicates that the Fermi surface is always large, in agreement with experimental findings.

We finally address ourselves to the temperature dependence of the Hall angle $\cot \theta_{H}$, which in the underdoped regime shows a $T^{2}$ behavior for a large variety of HTS, sometimes extending up to 400 K. In Fig.6 we plot our calculated Hall angle as a function of temperature for three different doping levels. We notice that deep inside the underdoped regime we do indeed find a $T^{2}$ behavior while with increased doping, going from $\Delta_{B}=1.26$ to $\Delta_{B}=1.18$, the range of this $T^{2}$ behavior gets more and more restricted to low temperatures, in qualitative agreement with the experiments.

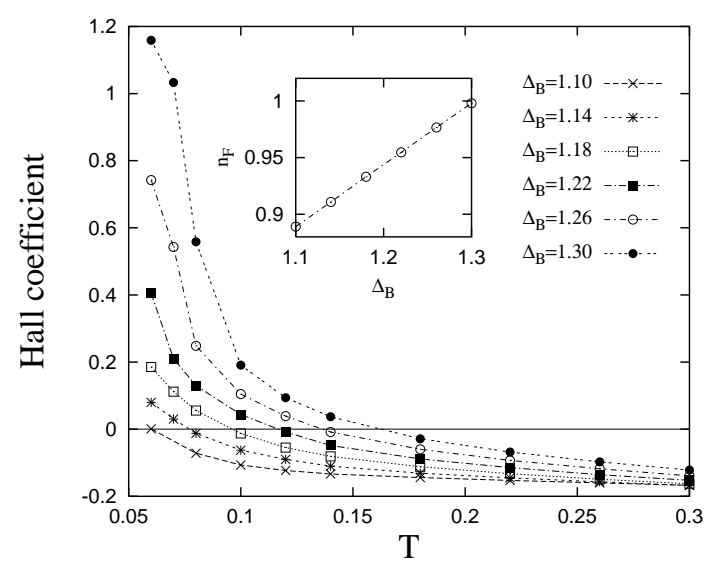

FIG. 5. Evolution of the Hall coefficient as a function of temperature for $n=1.5, g=0.2$ and different doping levels. The inset shows the variation of $n_{F}$ with $\Delta_{B}$ for $T=0.26$.

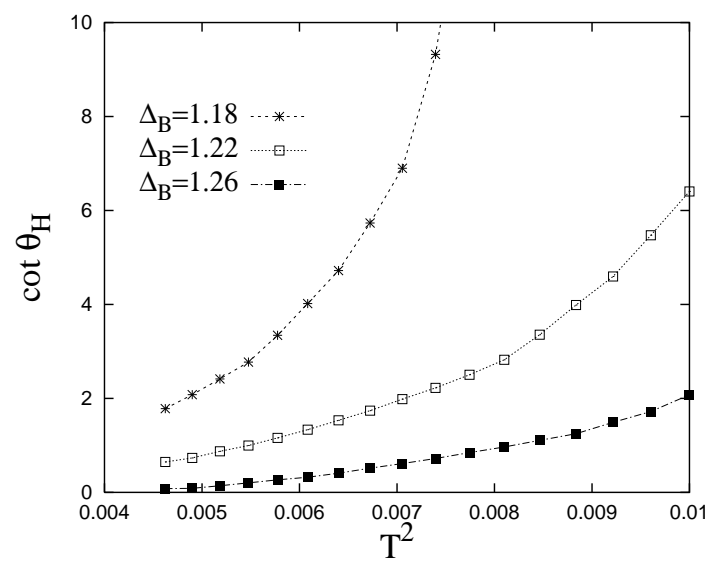

FIG. 6. Evolution of the Hall angle as a function of temperature for $n=1.5, g=0.2$ and different doping levels. 


\section{CONCLUSIONS}

The non-Fermi liquid properties of the cuprate HTS seem now to be established. Under these circumstances the evaluation of transport coefficients, such as the ones discussed here, can no longer be based on the standard Boltzmann-type relaxation time approach. The transport coefficients are no longer determined exclusively by scattering processes in the immediate vicinity of the Fermi surface, as is the case for standard Fermi liquids. On the contrary, scattering processes cover a wide regime in frequency and wave vector, in accordance with the experimental evidence for very broadened single-particle spectral functions. In this paper we have examined the question to what extent the anomalous properties of the Hall coefficient $R_{H}$ can be linked to the anomalous singleparticle properties and in particular to the pseudogap features of them. Our main result is a strongly temperature dependent $R_{H}$ which, for low temperatures, is positive (hole-like charge carriers) and scales with the number of holes as measured from the half-filled band case. As the temperature is increased, $R_{H}$ rapidly drops and saturates at very small negative values for temperatures above that where the pseudogap opens. Correspondingly, Fermi liquid properties begin to be recovered. Since we do not take into account electronic correlations, this result is what one should expect. Taking account of correlations should alter this result as far as the doping dependence of $R_{H}$ is concerned and should make $R_{H}$ scale with the number of holes (measured from the half-filled band case) even for high temperatures. This, however, requires a treatment of correlations and pair fluctuations on the same footing, which is beyond the present study. Finally, a $T^{2}$ behavior of the Hall angle is obtained, but exclusively for very low doped systems.

\section{ACKNOWLEDGEMENTS}

We would like to thank T. Domanski and K. Matho for stimulating discussions.

[1] H. Takagi, T. Ido, S. Ishibashi, M. Uota, S. Uchida, and Y. Tokura, Phys. Rev. B 40, 2254 (1989); T. Nishikawa, J. Takeda, and M. Sato, J. Phys. Soc. Japan 62, 2568 (1993); A. Carrington, D.J.C. Walker, A.P. Mackenzie, and J.R. Cooper, Phys. Rev. B 48, 13051 (1993); H.Y.
Hwang, B. Batlogg, H. Takagi, H.L. Kao, J. Kwo, R.J. Cava, J.J. Krajewski, and W.F. Peck Jr., Phys. Rev. Lett. 72, 2636 (1994).

[2] P.W. Anderson, Phys. Rev. Lett. 67, 2092 (1991); N.H. Hussey, J.R. Cooper, J.M. Wheatley, I.R. Fisher, A. Carrington, A.P. Mackenzie, C.T. Lin, and O. Milat, Phys. Rev. Lett. 76, 122 (1996).

[3] T. Pruschke, M. Jarrell and J. Freericks, Adv. Phys. 44 187 (1995).

[4] E. Lange and G. Kotliar, Phys. Rev. B 59, 1800 (1999).

[5] B.S. Shastry, B.I. Shraiman and R.R.P. Singh, Phys. Rev. Lett. 70, 2004 (1993).

[6] J. Corson, R. Mallozzi, J.N. Eckstein, I. Bozovic, and J. Orenstein, Nature 398, 221 (1999).

[7] M. Randeria, Proc. Int. School of Physics "Enrico Fermi", Course CXXXVI, eds. G. Iadonisi, R. Schrieffer and M.L. Chiofalo (IOS Press, Amsterdam, 1998) pp. 5375; O. Tchernyshyov, Phys. Rev. B 56, 3372 (1997); R. Micnas, M.H. Pedersen, S. Schafroth, T. Schneider, J.J. Rodríguez Núñez, and H. Beck, Phys. Rev. B 52, 16223 (1995); B. Janko, J. Mali and K. Levin, Phys. Rev. B 56, R11407 (1997); M. Letz and R. J. Gooding, J. Phys.: Condens. Matter 10, 6931 (1998).

[8] J. Ranninger and S. Robaszkiewicz, Physica B 135, 468 (1998).

[9] J. Ranninger, J.-M. Robin and M. Eschrig, Phys. Rev. Lett. 74, 4027 (1995), J. Ranninger and J.-M. Robin, Solid State Commun. 98, 559 (1996), J. Ranninger and J.-M. Robin, Phys. Rev. B 53 R11961 (1996).

[10] T. Domanski, J. Ranninger and J.-M. Robin, Solid State Commun. 105, 473 (1998).

[11] see for instance: A. Georges, G. Kotliar, W. Krauth and M. Rozenberg, Rev. Mod. Phys. 68, 13 (1996); F. Gebhard, "The Mott Metal-Insulator Transition" (Springer Verlag, berlin, 1997).

[12] N.E. Bickers, Rev. Mod. Phys. 59, 845 (1987).

[13] J.-M. Robin, A. Romano and J. Ranninger, Phys. Rev. Lett. 81, 2756 (1998).

[14] P. Lombardo, J. Schmalian, M. Avignon, and K.-H. Bennemann, Phys. Rev. B 54, 5317 (1997).

[15] T. Schork and S. Blawid, Phys. Rev. B 56, 6559 (1997).

[16] T. Schork, S. Blawid, and J. Igarashi, Phys. Rev. B 59, 9888 (1999).

[17] M. Randeria, G. Jennings, H. Ding, J.-C. Campuzano, A. Bellman, T. Yokoya, T. Takahashi, H. KatayamaYoshida, T. Mochiku, and K. Kadowaki, Phys. Rev. Lett. 74, 4951 (1995).

[18] A. Ino, C. Kim, T. Mizokawa, Z.-X. Shen, A Fujimori, M. Takaba, K. Tamasaku, H. Eisaki, and S. Uchida, J. Phys. Soc. Japan 68, 1496 (1999).

[19] T. Chien, Z.Z. Wang, and N.P. Ong, Phys. Rev. Lett. 67, 2088 (1991); G. Xiao, P. Xiong, and M.Z. Cieplak, Phys. Rev. B 46, 8687 (1992); B. Bucher, P. Steiner, J. Karpinski, E. Kaldis, and P. Wachter, Phys. Rev. Lett. 70, 2012 (1993); J.M. Harris, H. Wu, N.P. Ong, R.L. Meng, and C.W. Chu, Phys. Rev. B 50, 3246 (1994). 